\title{
Economic Analysis of Micro Hydel Plant-A Case Study
}

\author{
Amandeep Kaur ${ }^{1}$, Navdeep Kaur Brar ${ }^{2}$ \\ ${ }^{1}$ Baba Banda Singh Bahadur Engineering College Fatehgarh Sahib, India
}

${ }^{2}$ Assistant Professor Department of Electrical Engineering, Baba Banda Singh Bahadur Engineering College Fatehgarh Sahib, India

\begin{abstract}
A reliable, economical and impregnable supply of energy is consequential for economic development. This has been true for the past and present and will remain authentic for the future. However, over time, changes have taken and will take place with regard to energy utilization, both with regard to the amount as well as with regard to the type of energy utilized. Many factors have played a role in bringing these vicissitudes. Availability, security of supplies, price, ease of handling and use, external factors like technological development, introduction of subsidies, environmental constraints and legislation are some of these factors. This research paper is an in-depth scenario and economic analysis of the micro hydro energy in Punjab.
\end{abstract}

Keywords: micro hydel plant, payback period, economic analysis

\section{Introduction}

The hydro power plants with engendered power less than 10 MW are becoming more captivating considering both technical - economic aspects and environmental issues. Supplementally, this type of energy production is environmentally amicable, contributes toward gas emission and global warming reductions and can be habituated to regulate the watercourses.

Hydro power plants according to their installed power can be "small power plants" when they engender from 2 to $10 \mathrm{MW}$, "mini" when between 2 and $0.5 \mathrm{MW}$ and "micro" when less than 0.5 MW. From the standpoint of water height, hydro plants can be relegated as "low" when the height of fall is from 2 to $20 \mathrm{~m}$, "average" for the range from 20 to $150 \mathrm{~m}$, and "high" when it emanates over $150 \mathrm{~m}[1]$. Micro hydro is the well-known principle of utilizing water to drive a turbine and generate electricity; however micro hydro is executed on a much diminutive scale including private residences and businesses. Not only micro hydro is a non-polluting energy source, but also it is much more efficient than the burning of fossil fuels for electricity generation. In deference to coal burning, the most mundane energy source, micro hydro power is greatly more efficient. Efficiency of micro hydro units range $60 \%$ to $90 \%$ while modern coal burning units are $43 \%$ to $60 \%$ efficient [2].

This case study is predicated on a micro-hydro power plant with an average height of water of $2.84 \mathrm{~m}$, an average water flow rate of $29.94 \mathrm{~m} 3 / \mathrm{s}$ and a global efficiency of $90 \%$.

\section{Economic Analysis}

The performed economic analysis exhibits the expenses and revenues of the system and enables one to estimate the economic indicators required to state the viability of the solution.

The system had the initial investment of 14 crores, including costs of building, electrical parts and mechanical parts, as summarized in Table 1.

Table 1: Investment Costs

\begin{tabular}{|c|c|}
\hline Project/Preliminary studies & 1 crore \\
\hline Grid Connection & 1 crore \\
\hline Administrative costs & 0.5 crore \\
\hline Civil work & 5 crore \\
\hline Mechanical parts & 2.5 crore \\
\hline Turbine/generator and accessories & 2.5 crore \\
\hline Electric parts & 0.5 crore \\
\hline Total & 14 crore \\
\hline
\end{tabular}

Investment of the plant $=14$ crore $=$ Rs -140000000

Generation of plant $=1 \mathrm{MW}=1000 \mathrm{KW}$

Number of units produced per day $=1000 * 24$

$=24000 \mathrm{KWH}$

$=24000$ units

Number of units used for plant auxiliaries $=960$

Therefore approximately 23000 units are being sold to PSPCL.

Rate of one unit given by PSPCL= Rs 3.25

So, income per day $=23000 * 3.25$

$=$ Rs 74880

Income per year $=74880 * 365$ days

$=$ Rs 27331200

Operation cost $=10$ paisa per unit

Operation cost for 24000 units $=24000 * 0.1=$ Rs 2400 per day

Operation cost per year $=2400 * 365=$ Rs 876000

Maintenance cost $=33$ paisa per unit

Maintenance cost for 24000 units $=24000 * 0.33=$ Rs 7920 per day

Maintenance cost per year $=7920 * 365=$ Rs 2890800

Cash flow $=$ Income - (operation cost + maintenance cost $)$

$=27331200-(876000+2890800)$

$=$ Rs 23564400

Payback $=$ Investment + cash flow

$=-140000000+23564400$

$=-116435600$

\section{Volume 5 Issue 4, April 2016}




\section{International Journal of Science and Research (IJSR) \\ ISSN (Online): 2319-7064 \\ Index Copernicus Value (2013): 6.14 | Impact Factor (2015): 6.391}

Analysis of the plant has been done for 25 years

Case 1: Taking the constant income for all 25 years

Table 2

\begin{tabular}{|c|c|c|c|c|c|c|}
\hline Year & 0 & 1 & 2 & 3 & 4 & 5 \\
\hline Income & & 27331200 & 27331200 & 27331200 & 27331200 & 27331200 \\
\hline Operation costs (Year rate:5\%) & & -876000 & -919800 & -965790 & -1014080 & -1521120 \\
\hline Maintenance costs (Year rate:3\%) & & -2890800 & -2977524 & -3066850 & -3986905 & -4106512 \\
\hline Investment & -140000000 & & & & & \\
\hline Cash flow & & 23564400 & 23433876 & 23298560 & 22330215 & 21703568 \\
\hline Payback & & -116435600 & -93001724 & -69703164 & -47372949 & -25669381 \\
\hline IRR(25years) & & & & $-27.92 \%$ & $-14.803 \%$ & $-6.478 \%$ \\
\hline
\end{tabular}

\begin{tabular}{|c|c|c|c|c|c|c|}
\hline & & 6 & 7 & 8 & 9 & 10 \\
\hline Year & & 27331200 & 27331200 & 27331200 & 27331200 & 27331200 \\
\hline Income & & -1597176 & -1677035 & -1760887 & -1848931 & -1941378 \\
\hline Operation costs (Year rate:5\%) & & -4229707 & -126891 & -4487296 & -4621915 & -4760572 \\
\hline Maintenance costs (Year rate:3\%) & & & & & & \\
\hline Investment & & 21504317 & 21297567 & 21083017 & 20860354 & 20629250 \\
\hline Cash flow & & -4165064 & 17132503 & 38215520 & 59075874 & 79705124 \\
\hline Payback & & $-0.871 \%$ & $3.034 \%$ & $5.835 \%$ & $7.893 \%$ & $9.437 \%$ \\
\hline IRR(25years) & &
\end{tabular}

\begin{tabular}{|c|c|c|c|c|c|c|}
\hline Year & & 11 & 12 & 13 & 14 & 15 \\
\hline Income & & 27331200 & 27331200 & 27331200 & 27331200 & 27331200 \\
\hline Operation costs (Year rate:5\%) & & -2038447 & -2140369 & -2247387 & -2359756 & -2477744 \\
\hline Maintenance costs (Year rate:3\%) & & -4903389 & -5050410 & -5201922 & -5357980 & -5518719 \\
\hline Investment & & & & & & \\
\hline Cash flow & & 20389364 & 20140421 & 19881891 & 19613464 & 19334737 \\
\hline Payback & & 100094488 & 120234909 & 140116800 & 159730264 & 179065001 \\
\hline IRR(25years) & & $10.616 \%$ & $11.528 \%$ & $12.243 \%$ & $12.809 \%$ & $13.261 \%$ \\
\hline
\end{tabular}

\begin{tabular}{|c|c|c|c|c|c|c|}
\hline Year & & 16 & 17 & 18 & 19 & 20 \\
\hline Income & & 27331200 & 27331200 & 27331200 & 27331200 & 27331200 \\
\hline Operation costs (Year rate:5\%) & & -2601631 & -2731713 & -2868299 & -3011714 & -3162300 \\
\hline Maintenance costs (Year rate:3\%) & & -5684281 & -5854809 & -6030453 & -6211367 & -6397708 \\
\hline Investment & & & & & & \\
\hline Cash flow & & 19045288 & 18744678 & 18432448 & 18108119 & 17771192 \\
\hline Payback & & 198110289 & 216854967 & 235287415 & 253395534 & 271166726 \\
\hline IRR(25years) & & $13.625 \%$ & $13.920 \%$ & $14.160 \%$ & $14.356 \%$ & $14.517 \%$ \\
\hline
\end{tabular}

\begin{tabular}{|l|l|l|l|l|l|l|}
\hline Year & & \multicolumn{1}{|c|}{21} & \multicolumn{1}{c|}{22} & \multicolumn{1}{c|}{23} & \multicolumn{1}{c|}{24} & \multicolumn{1}{c|}{25} \\
\hline Income & & 27331200 & 27331200 & 27331200 & 27331200 & 27331200 \\
\hline Operation costs(Year rate:5\%) & & -3320415 & -3486436 & -3660758 & -3843796 & -4035986 \\
\hline Maintenance costs(Year rate:3\%) & & -6589639 & -6787328 & -6990948 & -7200678 & -7560712 \\
\hline Investment & & & & & & \\
\hline Cash flow & & 17421146 & 17057436 & 16679494 & 16286726 & 15734502 \\
\hline Payback & & 288587872 & 305645308 & 322324802 & 338611528 & 354346030 \\
\hline IRR(25years) & $14.98 \%$ & $14.649 \%$ & $14.7590 \%$ & $14.8496 \%$ & $14.924 \%$ & $14.98643 \%$ \\
\hline
\end{tabular}

CASE 2: Increasing income by year rate of $2 \%$

Table 3

\begin{tabular}{|c|c|c|c|c|c|c|}
\hline Year & 0 & 1 & 2 & 3 & 4 & 5 \\
\hline Income & & 27331200 & 27877824 & 28435380 & 29004087 & 29584169 \\
\hline Operation costs (Year rate:5\%) & & -876000 & -919800 & -965790 & -1014080 & -1521120 \\
\hline Maintenance costs (Year rate:3\%) & & -2890800 & -2977524 & -3066850 & -3986905 & -4106512 \\
\hline Investment & -140000000 & & & & & \\
\hline Cash flow & & 23564400 & 23980500 & 24402740 & 24003102 & 23956537 \\
\hline Payback & & -116435600 & -92455100 & -68052360 & -44049258 & -20092721 \\
\hline IRR(25years) & & & & $-27.00 \%$ & $-13.530 \%$ & $-4.9416 \%$ \\
\hline
\end{tabular}

\section{Volume 5 Issue 4, April 2016}


International Journal of Science and Research (IJSR)
ISSN (Online): 2319-7064

Index Copernicus Value (2013): 6.14 | Impact Factor (2015): 6.391

\begin{tabular}{|c|c|c|c|c|c|c|}
\hline Year & & 6 & 7 & 8 & 9 & 10 \\
\hline Income & & 30175852 & 30779369 & 31394956 & 32022855 & 32663312 \\
\hline Operation costs(Year rate:5\%) & & -1597176 & -1677035 & -1760887 & -1848931 & -1941378 \\
\hline Maintenance costs(Year rate:3\%) & & -4229707 & -4356598 & -4487296 & -4621915 & -4760572 \\
\hline Investment & & & & & & \\
\hline Cash flow & & 24348969 & 24745736 & 25146773 & 25552009 & 25961362 \\
\hline Payback & & 4256248 & 29001984 & 54148757 & 79700766 & 105662128 \\
\hline IRR(25years) & & $0.85947 \%$ & $4.9112 \%$ & $7.8255 \%$ & $9.9742 \%$ & $11.5917 \%$ \\
\hline
\end{tabular}

\begin{tabular}{|c|c|c|c|c|c|c|}
\hline Year & & 11 & 12 & 13 & 14 & 15 \\
\hline Income & & 33316578 & 33982909 & 34662567 & 35355818 & 36062934 \\
\hline Operation costs(Year rate:5\%) & & -2038447 & -2140369 & -2247387 & -2359756 & -2477744 \\
\hline Maintenance costs(Year rate:3\%) & & -4903389 & -5050410 & -5201922 & -5357980 & -5518719 \\
\hline Investment & & & & & & \\
\hline Cash flow & & 26374742 & 26792130 & 27213258 & 27638082 & 28066471 \\
\hline Payback & & 132036870 & 158829000 & 186042258 & 213680340 & 241746811 \\
\hline IRR(25years) & & $12.831 \%$ & $13.7936 \%$ & $14.552 \%$ & $15.1547 \%$ & $15.6390 \%$ \\
\hline
\end{tabular}

\begin{tabular}{|l|l|l|l|l|l|l|}
\hline Year & & \multicolumn{1}{|c|}{16} & \multicolumn{1}{|c|}{17} & \multicolumn{1}{c|}{18} & \multicolumn{1}{c|}{19} & \multicolumn{1}{c|}{20} \\
\hline Income & & 36784193 & 37519877 & 38270275 & 39035681 & 39816395 \\
\hline Operation costs(Year rate:5\%) & & -2601631 & -2731713 & -2868299 & -3011714 & -3162300 \\
\hline Maintenance costs(Year rate:3\%) & & -5684281 & -5854809 & -6030453 & -6211367 & -6397708 \\
\hline Investment & & & & & & \\
\hline Cash flow & & 28498281 & 28933355 & 29371523 & 29182600 & 30256387 \\
\hline Payback & & 270245092 & 299178447 & 328549970 & 358362570 & 388618957 \\
\hline IRR(25years) & & $15.6390 \%$ & $16.0309 \%$ & $16.3504 \%$ & $16.8283 \%$ & $17.0072 \%$ \\
\hline
\end{tabular}

\begin{tabular}{|l|l|l|l|l|l|l|}
\hline Year & & \multicolumn{1}{|c|}{21} & \multicolumn{1}{c|}{22} & \multicolumn{1}{c|}{23} & \multicolumn{1}{c|}{24} & \multicolumn{1}{c|}{25} \\
\hline Income & & 40612723 & 41424977 & 42253477 & 43098547 & 43960518 \\
\hline Operation costs(Year rate:5\%) & & -3320415 & -3486436 & -3660758 & -3880403 & -4074423 \\
\hline Maintenance costs(Year rate:3\%) & & -6589639 & -6787328 & -6990948 & -7200676 & -7416696 \\
\hline Investment & & & & & & \\
\hline Cash flow & & 30702669 & 31151213 & 31601771 & 32017468 & 32469399 \\
\hline Payback & & 419321626 & 450472839 & 482074610 & 514092078 & 546561477 \\
\hline IRR(25years) & $17.54 \%$ & $17.2801 \%$ & $17.28 \%$ & $17.38407 \%$ & $17.471 \%$ & $17.545 \%$ \\
\hline
\end{tabular}

CASE 3: Constant from year 1 to 5; Decreasing year rate: 2\% - from year 6 to year 25.

Table 4

\begin{tabular}{|c|c|c|c|c|c|c|}
\hline Year & 0 & 1 & 2 & 3 & 4 & 5 \\
\hline Income & & 25623000 & 25623000 & 25623000 & 25623000 & 25623000 \\
\hline Operation costs(Year rate:5\%) & & -876000 & -963600 & -1059960 & -1165956 & -1282552 \\
\hline Maintenance costs(Year rate:3\%) & & -2890800 & -3064248 & -3248103 & -3442989 & -3649568 \\
\hline Investment & -140000000 & & & & & \\
\hline Cash flow & & 22644600 & 21595152 & 21314937 & 21014055 & 20690880 \\
\hline Payback & & -117355400 & -95760248 & -74445311 & -53431256 & -32740376 \\
\hline IRR(25years) & & & & $-30.324 \%$ & $-16.959 \%$ & $-8.3738 \%$ \\
\hline
\end{tabular}

\begin{tabular}{|l|l|l|l|l|l|l|}
\hline Year & & 6 & 7 & 8 & 9 & 10 \\
\hline Income & & 25110540 & 24608329 & 24116162 & 23633839 & 23161162 \\
\hline Operation costs(Year rate:5\%) & & -1410807 & -1551888 & -1707077 & -1877785 & -2065564 \\
\hline Maintenance costs(Year rate:3\%) & & -3868542 & -4100655 & -4346694 & -4607496 & -4745721 \\
\hline Investment & & & & & & \\
\hline Cash flow & & 19831191 & 18955786 & 18062391 & 17148558 & 16349877 \\
\hline Payback & & -12909185 & 6046601 & 24108992 & 41257550 & 57607427 \\
\hline IRR(25years) & & $-2.7467 \%$ & $1.0974 \%$ & $3.80853 \%$ & $5.7686 \%$ & $7.2245 \%$ \\
\hline
\end{tabular}

\section{Volume 5 Issue 4, April 2016}


International Journal of Science and Research (IJSR)

ISSN (Online): 2319-7064

Index Copernicus Value (2013): 6.14 | Impact Factor (2015): 6.391

\begin{tabular}{|l|l|l|l|l|l|l|}
\hline Year & & 11 & 12 & 13 & 14 & 15 \\
\hline Income & & 22697939 & 22243980 & 21799100 & 21363118 & 20935856 \\
\hline Operation costs(Year rate:5\%) & & -2272120 & -2499332 & -2749265 & -3024192 & -3326611 \\
\hline Maintenance costs(Year rate:3\%) & & -5030464 & -5332292 & -5652230 & -5991364 & -6350846 \\
\hline Investment & & & & & & \\
\hline Cash flow & & 15395355 & 14412356 & 13397605 & 12347562 & 11258399 \\
\hline Payback & & 73002782 & 87415138 & 100812743 & 113160305 & 124418704 \\
\hline IRR(25years) & & $8.31347 \%$ & $9.1369 \%$ & $9.7646 \%$ & $10.2449 \%$ & $10.6126 \%$ \\
\hline
\end{tabular}

\begin{tabular}{|l|l|l|l|l|l|l|}
\hline Year & & \multicolumn{1}{|c|}{16} & \multicolumn{1}{c|}{17} & \multicolumn{1}{c|}{18} & \multicolumn{1}{c|}{19} & \multicolumn{1}{c|}{20} \\
\hline Income & & 20517139 & 20106796 & 19704660 & 19310567 & 18924356 \\
\hline Operation costs(Year rate:5\%) & & -3659272 & -4025199 & -4427719 & -4870491 & -5357540 \\
\hline Maintenance costs(Year rate:3\%) & & -6731897 & -7135811 & -7563960 & -8017798 & -8498866 \\
\hline Investment & & & & & & \\
\hline Cash flow & & 10125970 & 8945786 & 7712981 & 6422278 & 5067950 \\
\hline Payback & & 123286275 & 132232061 & 139945042 & 146367320 & 151435270 \\
\hline IRR(25years) & & $10.8932 \%$ & $11.1054 \%$ & $11.2633 \%$ & $11.3777 \%$ & $11.4567 \%$ \\
\hline
\end{tabular}

\begin{tabular}{|l|l|l|l|l|l|l|}
\hline Year & & \multicolumn{1}{|c|}{21} & \multicolumn{1}{c|}{22} & \multicolumn{1}{c|}{23} & \multicolumn{1}{c|}{24} & \multicolumn{1}{c|}{25} \\
\hline Income & & 18545869 & 18174952 & 17811453 & 17455224 & 17106119 \\
\hline Operation costs(Year rate:5\%) & & -5893294 & -6482623 & -7130885 & -7843974 & -8628371 \\
\hline Maintenance costs(Year rate:3\%) & & -9008798 & -9549326 & -10122286 & -10729623 & -11373400 \\
\hline Investment & & & & & & \\
\hline Cash flow & & 3643777 & 2143003 & 558282 & -1118373 & -2895652 \\
\hline Payback & & 155079047 & 157222050 & 157780332 & 156661959 & 153766307 \\
\hline IRR(25years) & $11.51 \%$ & $11.50678 \%$ & $11.5328 \%$ & $11.5389 \%$ & $11.53781 \%$ & $11.51290 \%$ \\
\hline
\end{tabular}

\section{Results and Discussion}

The results presented in Table 2, were obtained using present-day conditions applicable to micro-hydro power plants in Punjab. Under a 25-year scenario these conditions will transmute. Mainly, the following situations could occur:

- Decrease in the feed-in tariff;

- Increase in the operation costs and maintenance costs;

- Lower water flow rate.

Table 3 illustrates the results if the income increases by $2 \%$ every year. Taking into consideration the factors mentioned for Table 2, the following scenario is considered which is illustrated in Table 4:

- Constant feed-in tariff (present-day value) during the first 5 years;

- After year 5, the feed-in-tariff decreases 2\%/year;

- Operation costs increase 10\%/year (rate doubled);

- Maintaining costs increase 6\%/year (rate doubled);

- The efficiency of the group turbine-generator decreases to $75 \%$.

The analysis presented in table 3 is economically captivating with its IRR of $17.54 \%$ and payback within 6 years.

For the worst case scenario (Table 4), these indicators decrease, with an IRR of $11.51 \%$ and payback within 7 years.

Another economic indicator for the three scenarios is the Net Present Value, which provides the following values:

- Table 2 scenario, NPV=102333927.8 rupees

- Table 3 scenario, NPV= 5982460 rupees

- Table 4 scenario, NPV=42075352.3 rupees
Net Present Value (NPV) is the quantification of an investment's profitability.

\section{Conclusion}

This paper presents an overview of the sundry economic issues of the micro hydro power plant .Economic aspects of the power plant are analyzed by calculating its payback period. The cost of the MHP is site categorical and varies remarkably depending on the remoteness of the site and physical features of its major components, namely, civil works, generating equipments and transmission/distribution lines. The performed analysis limpidly points out the vigorous and impotent aspects considering economic issues.

As a final remark it is to verbally express that these micro hydro power plants are very efficacious in meeting energy demand and contribute to the reduction of dependence on polluting power plants. Due to their typical advantages, more and more micro hydro power plants should be established to regulate the water courses.

\section{References}

[1] A.Roque, D. M. Sousa, C. Casimiro and E. Margato, "Technical and economic analysis of a micro hydro plant - a case study," Energy Market (EEM), 2010 7th International Conference on the European, Madrid, 2010, pp. 1-6.

[2] M.A.Wazed and Shamsuddin Ahmed, Micro Hydro Energy Resources in Bangladesh: A Review.

[3] Arun Varughese, Prawin Angel Michael, Electrical Characteristics of Micro-Hydro Power Plant Proposed in Valara Waterfall" International Journal of Innovative 
Technology and Exploring Engineering (IJITEE), Vol. 2, Issue 2, January 2013.

[4] Jahidul Islam Razan, Riasat Siam Islam, Rezaul Hasan,Samiul Hasan, and Fokhrul Islam, -A Comprehensive Study of Micro-Hydropower Plant and Its Potential in Bangladesh" ISRN Renewable Energy, 2012.

[5] M. A. M. Badrin, "Micro Hydro Power," Malaysia, 2009.Japan International Coorporation Agency, Manuals and Guidelines for Micro-hydropower Development in Rural Electrification, Japan: Department of Energy (DOE), 2009. 